\title{
RECOGNIZING MANIFESTATIONS OF POSTTRAUMATIC STRESS DISORDER IN PATIENTS WITH TRAUMATIC BRAIN INJURY
}

\author{
ERIC B. LARSON AND HEATHER G. BELANGER
}

\section{BACKGROUND}

\section{Definition}

Psychological trauma and traumatic brain injury (TBI) are both historical events that must be distinguished from their consequences. Not all psychological trauma results in persistent distress and not all injuries result in persistent disability or psychiatric complications. One syndrome that may persist after exposure to a traumatic event is posttraumatic stress disorder"(PTSD), a constellation of emotional, behavioral, and cognitive symptoms that may follow exposure to significant threat to life, serious physical injury, or sexual violence. Following moderate to severe TBI, persistent sequelae are common and PTSD is sometimes confused with other psychiatric disorders associated with injury such as adjustment disorder or personality change due to TBI. Following mild TBI, a full recovery is typical but in those with persistent psychiatric complications, differential diagnosis can be particularly complicated. ${ }^{1}$ This chapter offers a brief summary of issues related to assessment and management of PTSD in these populations.

\section{Diagnostic Criteria}

Problems from each of the following five categories must be observed for a diagnosis of PTSD2:

History of exposure to trauma

Intrusion of trauma-related symptoms after the event

Avoidant behavior

- Dysfunctional changes in cognition and mood associated with trauma

Increased autonomic arousal

Diagnosis further requires that duration of symptoms is more than 1 month, and that these symptoms result in significant distress or functional impairment.

\section{Epidemiology}

In the general population, lifetime prevalence of PTSD is $7.8 \%$. In patients with mild TBI (MTBI), estimates of prevalence range from $10 \%$ to $27 \%$. Prevalence of PTSD has been shown to be the same among MTBI patients as it is among patients with other traumatic injuries. ${ }^{3}$ In patients with severe TBI, the best estimate of prevalence is 3\%, although selfreport of symptoms is much higher. ${ }^{4}$

The full reference list appears in the digital product found on http:/ / connect.springerpub.com/content/book/978-0-8261-4768-4/part/part02/chapter/ch18 


\section{Etiology}

\section{A Behavioral Account}

Most forms of anxiety are a result of appraisal of an impending (future) threat. PTSD involves processing a past trauma as a current threat, possibly because of activation of implicit memories of the traumatic events. Discrimination between current experience and past implicit memories may be more difficult because the latter are more vaguely defined than are explicit memories. ${ }^{5}$

\section{A Neurobiological Account}

The implicit learning involved in PTSD may be mediated by neural circuits that are characterized by:

Inadequate frontal inhibition (associated with inability to suppress attention to stimuli related to trauma)

- Excessive amygdala response (associated with conditioned fear and reactivity to potential threats)

- Compromised hippocampus function (associated with deficient ability to distinguish safe and unsafe environments) ${ }^{6}$

\section{Pathophysiology}

Inconsistent evidence of atrophy in the hippocampus and in the anterior cingulate cortex has been reported in structural imaging studies of PTSD patients. Some have proposed these are stress-induced changes, but twin studies suggest that reduced volume in these structures is a pretrauma vulnerability factor. ${ }^{7}$ Similarly, comparisons of structural, perfusion, and diffusion magnetic resonance imaging (MRI) data in 17 veterans with PTSD and in 15 age-matched veterans without PTSD showed increased regional cerebral blood flow in the right parietal and superior temporal cortices, and reduced functional anisotropy in white matter regions near the anterior cingulate, prefrontal lobe, and the posterior angular gyrus. As in previous imaging studies, it was concluded that these abnormalities may be the result of PTSD or may be risk factors that cause individuals to be predisposed to the disorder. ${ }^{8}$

\section{DIAGNOSIS}

\section{Risk Factors}

Knowledge of characteristics that leave patients vulnerable to PTSD can help determine if that disorder is present in a TBI survivor with an ambiguous clinical presentation.

- Pretrauma risk factors: Sex and marital status are the strongest demographic predictors of PTSD. Women and previously married (e.g., divorced or widowed) individuals have the highest risk for PTSD. ${ }^{9}$

- Trauma-related risk factors: For men, the highest risk for PTSD is for those who have been in combat and for those who have witnessed someone being killed or severely injured. For women, the highest risk is associated with rape or sexual molestation.

- Posttrauma risk factors: A lack of subsequent social support and experience of additional life stressors are both stronger predictors of PTSD than pre-trauma risk factors. ${ }^{10}$

- Poor cognitive function: ${ }^{11}$ A pretrauma cognitive deficit may leave an individual less able to cope with stressors, which may make them more vulnerable to PTSD. 
TBI patients are at increased risk for PTSD at 6 months after injuries if they experienced acute stress disorder after their injuries, if they exhibited symptoms of depression and anxiety within 1 week of injuries, if they have previous histories of psychiatric disorders, or if they had memories of the traumatic events. ${ }^{12}$

\section{Clinical Presentation}

Both people with PTSD and those with a history of TBI may complain of noise sensitivity, fatigue, anxiety, insomnia, poor concentration, poor memory, and irritability. The presence of these symptoms alone is not diagnostic of either disorder because all have high base rates in the general population as well.

\section{Symptoms}

Intrusion symptoms

- Recurrent involuntary, intrusive distressing recollections of the traumatic event. Such memories are sudden, unwanted, and disruptive to one's activities

- Recurrent distressing dreams of the event (nightmares)

- Dissociative behavior in which one acts or feels like the event is recurring (e.g., flashbacks). This involves perception that the trauma is happening in the present and differs from remembering the traumatic event as a past occurrence. Contact with present reality is diminished and in extreme cases may be entirely lost.

- Intense psychological distress at exposure to trauma-related cues

- Physiological reactivity on exposure to trauma-related cues

- Persistent avoidance

- Efforts to avoid thoughts or feelings associated with the trauma

- Efforts to avoid activities or situations that arouse recollections of trauma (e.g., appointment cancellations, failed appointments, and tardiness to treatment sessions)

Maladaptive changes in mood and cognition

- Inability to recall an important aspect of the trauma, only if determined not to be because of posttraumatic amnesia

- Persistent and overgeneralized dysfunctional beliefs about oneself, other individuals, or the world

- Persistent and distorted thoughts of blame

- Markedly diminished interest in significant activities that are still available despite physical disability

- Feeling of detachment or estrangement from others

- Persistent inability to experience positive emotions

- Reckless or self-destructive behavior

Maladaptive changes in arousal and reactivity

- Difficulty falling or staying asleep

- Irritability or outbursts of anger

- Difficulty concentrating

- Hypervigilance-which does not necessarily exclude situations in which an individual's perception of threat is justified by actual danger in their environment

- Exaggerated startle response

\section{Evaluation}

- Symptom checklists: Questionnaires that rely on self-report like the PTSD Checklist for DSM-5 (PCL-5) ${ }^{13}$ require little time to complete ( 5 minutes or less) and may be used as screening measures but should not be used for diagnosis given their poor specificity. 
That is, instruments designed to assess symptom type and severity are not helpful in identifying the specific mechanism responsible out of the many disorders in the differential. Further, in TBI patients, self-report is notoriously inaccurate.

- Structured interviews: The "gold standard" for PTSD diagnosis, clinician interviews require extended time to complete (30-120 minutes). They also require formal training to ensure inter-rater reliability. Measures include the Clinician-Administered PTSD Scale for DSM-5 (CAPS-5) ${ }^{14}$ and the Structured Clinical Interview for DSM-5 Disorders-Clinician Version (SCID-5-CV). ${ }^{15}$

- Neuropsychological evaluation: Standardized psychological assessment provides a detailed description of the nature of cognitive impairment and emotional distress. However, in cases where it is unclear whether a patient has sustained an MTBI, identifying cognitive impairment does not assist with differential diagnosis, because such impairment can be seen in individuals with PTSD alone. ${ }^{16}$

\section{Controversies}

It has been suggested that TBI does not produce PTSD because the disturbance of consciousness that must occur in the former interferes with formation of memories of trauma, which is presumably the cause of symptoms in the latter. ${ }^{17}$ Although some evidence supports this conclusion, other studies show that PTSD exists in individuals who lost consciousness at the time of their injuries. ${ }^{12,18}$ The formation of implicit memories (that may not require clear consciousness at the time of trauma and that may exist in the absence of explicit recall) has been offered as an explanation for this counterintuitive finding.

\section{TREATMENT}

\section{Guiding Principles}

\section{Exposure and Avoidance}

Treatment that increases exposure to trauma-related stimuli in a supportive, controlled environment is effective at reducing symptoms. Avoidance of stimuli that provoke distress results in increased anxiety when those triggers can no longer be escaped.

\section{Medication and Cognition}

Some pharmacological interventions are effective at short-term management of anxiety but can result in iatrogenic cognitive impairment, which makes them bad choices for TBI survivors.

\section{Psychopharmacology}

Sertraline, paroxetine, fluoxetine, or venlafaxine are effective and are strongly recommended for people with PTSD who choose not to engage in or are unable to access trauma-focused psychotherapy. Divalproex, tiagabine, guanfacine, risperidone, benzodiazepines, ketamine, hydrocortisone, or D-cycloserine are all ineffective, harmful, or both for people with PTSD. Practice guidelines strongly recommend that practitioners avoid prescribing these agents. ${ }^{19}$

\section{Psychotherapy}

- For patients capable of participation, there is high strength of evidence of a medium to large magnitude benefit for the critical outcome of PTSD symptom reduction for 
Prolonged Exposure therapy. Similarly, there is moderate strength of evidence of a medium to large magnitude benefit for Cognitive Processing Therapy, Cognitive Therapy and Mixed Cognitive-Behavioral Therapy. ${ }^{20}$

- Therapy can be introduced early for patients with MTBI and later in the course of recovery for moderate to severe TBI patients. Memory deficits are a substantial obstacle to efficacy; in fact, such impairment may never resolve to the point that psychotherapy is possible.

- Referral to cognitive-behavioral specialists can be particularly helpful. Centralized referral databases are now offered (listed in the Additional Reading section).

\section{Treatment Controversies}

Eye Movement Desensitization and Reprocessing

An ongoing debate continues about the efficacy of eye movement desensitization and reprocessing (EMDR). Although several outcome studies support the use of this technique, many clinicians argue that it is effective because it includes elements of exposure therapy, and that there are no advantages to EMDR over traditional exposure therapy. ${ }^{21}$

\section{Additional Considerations}

\section{Disability Evaluations}

In veterans, a diagnosis of PTSD may be used to support claims of disability. Disabled veterans can receive disability income if they can substantiate these claims. Secondary monetary gains may result in many false disability claims.

\section{Personal Injury Cases}

Individuals who file personal injury lawsuits may argue that their injuries resulted in PTSD. Again, secondary gains may influence symptom reporting. Consider referral for evaluation by a PTSD specialist and/or neuropsychologist who can determine the extent to which this influence may result in symptom magnification or malingering.

\section{KEY POINTS}

- People with PTSD and those with a history of TBI may both complain of noise sensitivity, fatigue, anxiety, insomnia, poor concentration, poor memory, and irritability. The presence of these symptoms alone is not diagnostic of either disorder because all have high base rates in the general population as well.

- Structured interviews are the gold standard for PTSD diagnosis. Due to poor specificity, self-report measures should not be used for this purpose.

- Prolonged Exposure, Cognitive Processing Therapy, Cognitive Therapy and Mixed Cognitive-Behavioral Therapy have all been shown to have significant benefit in PTSD. Psychopharmacology can also have value, especially in individuals with memory impairment complicating response to psychotherapy.

\section{STUDY QUESTIONS}

1. PTSD is a psychiatric syndrome that may follow exposure to all of the following except:

a. significant threat to life

b. financial exploitation 

c. serious physical injury
d. sexual violence

2. Which of the following treatments has high strength of evidence of a medium to large magnitude benefit for PTSD symptom reduction?
a. Prolonged Exposure therapy
b. Avoidant behavior
c. Sedative hypnotics (e.g., benzodiazepines)
d. Cognitive processing therapy

3. Which of the following is an example of an intrusion symptom?
a. Efforts to avoid thoughts or feelings associated with the trauma
b. Persistent and distorted thoughts of blame
c. Recurrent distressing dreams of the event (nightmares)
d. Irritability or outbursts of anger

4. The gold standard for PTSD diagnosis is
a. Neuropsychological evaluation
b. Structured clinical interview
c. The PTSD Checklist for DSM-5 (PCL-5)
d. Polygraphy

5. Which of the following is the strongest risk factor for PTSD?
a. Female sex
b. Pretrauma divorced marital status
c. Pretrauma widowed marital status
d. Posttrauma lack of social support and experience of additional life stressors

\section{ADDITIONAL READING}

\section{Practice Guideline (Pocket Guide): http://www.healthquality.va.gov/ptsd/ptsd_poc2.pdf}

Forbes D, Bisson JI, Monson CM, Berliner L. Effective treatments for PTSD: Practice guidelines from the international society for traumatic stress studies. 3rd ed. Guilford Press; 2020.

Gill IL, Mullin S, Simpson J. Psychosocial and psychological factors associated with post-traumatic stress disorder following traumatic brain injury in adult civilian populations: a systematic review. Brain Inj. 2014;28(1):1-14. doi:10.3109/02699052.2013.851416

McAllister TW. Psychopharmacological issues in the treatment of TBI and PTSD. Clin Neuropsychol. 2009;23(8):1338-1367. doi:10.1080/13854040903277289

To find a cognitive behavioral therapist: https://www.findcbt.org/FAT

Vanderploeg RD, Belanger HG, Curtiss G. Mild traumatic brain injury and posttraumatic stress disorder and their associations with health symptoms. Arch Phys Med Rehabil. 2009;90(7):1084-1093. doi:10.1016/ j.apmr.2009.01.023

\section{ANSWERS TO STUDY QUESTIONS}

1. Correct answer: $b$

Posttraumatic stress disorder (PTSD), a constellation of emotional, behavioral, and cognitive symptoms that may follow exposure to significant threat to life, serious physical injury, or sexual violence. Although financial exploitation may accompany other forms of abuse, when it occurs in isolation it is not a recognized trigger of PTSD as defined in the DSM-5. 
Further Reading:

American Psychiatric Association. Diagnostic and Statistical Manual of Mental Disorders. 5th ed. American Psychiatric Association; 2013.

2. Correct answer: a

Avoidant behavior is a symptom of PTSD and not a treatment. Sedative hypnotics are specifically contraindicated due to harmful effects. A systematic review of outcome research showed that prolonged exposure therapy has a high strength of evidence of a medium to large magnitude benefit for PTSD symptom reduction, while cognitive processing therapy has moderate strength of evidence.

Further Reading:

American Psychological Association. Clinical practice guideline for the treatment of posttraumatic stress disorder (PTSD) in adults. Author; 2017.

Department of Veterans Affairs, Department of Defense. VAIDoD clinical practice guideline for the management of posttraumatic stress disorder and acute stress disorder. 2017. www.healthquality.va.gov/guidelines/MH/ptsd/VADoDPTSDCPGFinal.pdf

3. Correct answer: $c$

In the DSM-5, efforts to avoid thoughts or feelings are considered a form of avoidance, persistent and distorted thoughts of blame are classified as maladaptive changes in mood and cognition, and irritability is listed as a form of maladaptive change in arousal. Nightmares are considered an intrusion symptom.

Further Reading:

American Psychiatric Association. Diagnostic and Statistical Manual of Mental Disorders. 5th ed. Author; 2013.

4. Correct answer: $b$

Structured clinical diagnostic interviews are the gold standard against which other approaches (e.g., self-report questionnaires like the PCL-5) are evaluated.

Further Reading:

Spoont M, Arbisi P, Fu S, et al. Screening for Post-Traumatic Stress Disorder (PTSD) in Primary Care: A Systematic Review [Internet]. Department of Veterans Affairs (US); 2013.

5. Correct answer: $d$

A lack of subsequent social support and experience of additional life stressors are both stronger predictors of PTSD than pre-trauma risk factors.

Further Reading:

Brewin CR, Andrews B, Valentine JD. Meta-analysis of risk factors for posttraumatic stress disorder in trauma-exposed adults. J Consult Clin Psychol. 2000;68(5):748-766.

\section{REFERENCES}

The full reference list appears in the digital product found on http://connect .springerpub.com/content/book/978-0-8261-4768-4/part/part02/chapter/ch18 


\section{Digital Content Only}

1. Vasterling JJ, Jacob SN, Rasmusson A. Traumatic brain injury and posttraumatic stress disorder: conceptual, diagnostic, and therapeutic considerations in the context of co-occurrence. $J$ Neuropsychiatry Clin Neurosci. 2018;30(2):91-100. doi:10.1176/appi.neuropsych.17090180

2. American Psychiatric Association. Diagnostic and Statistical Manual of Mental Disorders. 5th ed. Author; 2013.

3. Kim E, Lauterbach EC, Reeve A, et al. Neuropsychiatric complications of traumatic brain injury: a critical review of the literature (a report by the ANPA Committee on Research). J Neuropsychiatry Clin Neurosci. 2007;19(2):106-127. doi:10.1176/jnp.2007.19.2.106

4. Sumpter RE, McMillan TM. Misdiagnosis of post-traumatic stress disorder following severe traumatic brain injury. Br J Psychiatry. 2005;186:423-426. doi:10.1192/bjp.186.5.423

5. Ehlers A, Clark DM. A cognitive model of posttraumatic stress disorder. Behav Res Ther. 2000;38(4):319-345. doi:10.1016/S0005-7967(99)00123-0

6. Sherin JE, Nemeroff CB. Post-traumatic stress disorder: the neurobiological impact of psychological trauma. Dialogues Clin Neurosci. 2011:13:263-278. doi:10.31887/DCNS.2011.13.2/jsherin

7. Gilbertson MW, Shenton ME, Ciszewski A, et al. Smaller hippocampal volume predicts pathologic vulnerability to psychological trauma. Nat Neurosci. 2002;5(11):1242-1247. doi:10.1038/ nn958

8. Schuff N, Zhang Y, Zhan W, et al. Patterns of altered cortical perfusion and diminished subcortical integrity in posttraumatic stress disorder: a MRI study. Neuroimage. 2011:54S1:S62-S68. doi:10.1016/j.neuroimage.2010.05.024

9. Kessler RC, Sonnega A, Bromet E, et al. Posttraumatic stress disorder in the National Comorbidity Survey. Arch Gen Psychiatry. 1995;52(12):1048-1060. doi:10.1001/archpsyc.1995.03950240066012

10. Brewin CR, Andrews B, Valentine JD. Meta-analysis of risk factors for posttraumatic stress disorder in trauma-exposed adults. J Consult Clin Psychol. 2000;68(5):748-766. doi:10.1037/0022-006X.68.5.748

11. Parslow RA, Jorm AF. Pretrauma and posttrauma neurocognitive functioning and PTSD symptoms in a community sample of young adults. Am J Psychiatry. 2007;164(3):509-515. doi:10.1176/ ajp.2007.164.3.509

12. Gil S, Caspi Y, Ben-Ari IZ, et al. Does memory of a traumatic event increase the risk for posttraumatic stress disorder in patients with traumatic brain injury? A prospective study. Am J Psychiatry. 2005;162(5):963-969. doi:10.1176/appi.ajp.162.5.963

13. Weathers FW, Litz BT, Keane TM, et al. The PTSD Checklist for DSM-5 (PCL-5). 2013. www.ptsd. va.gov

14. Weathers FW, Blake DD, Schnurr PP, et al. The Clinician-Administered PTSD Scale for DSM-5 (CAPS-5). 2013. www.ptsd.va.gov

15. First MB, Williams JBW, Karg RS. Structured Clinical Interview for DSM-5 Disorders - Clinician Version (SCID-5-CV). American Psychiatric Association Publishing; 2015.

16. Brenner LA, Ladley-O'Brien SE, Harwood JE, et al. An exploratory study of neuroimaging, neurologic, and neuropsychological findings in veterans with traumatic brain injury and/or posttraumatic stress disorder. Mil Med. 2009;174(4):347-352. doi:10.7205/MILMED-D-01-5808

17. Sbordone RJ, Liter JC. Mild traumatic brain injury does not produce post-traumatic stress disorder. Brain Inj. 1995;9(4):405-412. doi:10.3109/02699059509005780

18. Mayou RA, Black J, Bryant B. Unconsciousness, amnesia and psychiatric symptoms following road traffic accident injury. Br J Psychiatry. 2000;177:540-545. doi:10.1192/bjp.177.6.540

19. Department of Veterans Affairs, Department of Defense. VA/DoD clinical practice guideline for the management of posttraumatic stress disorder and acute stress disorder. 2017. www.healthquality. va.gov/guidelines/MH/ptsd/VADoDPTSDCPGFinal.pdf

20. American Psychological Association. Clinical practice guideline for the treatment of posttraumatic stress disorder (PTSD) in adults. Author; 2017.

21. Albright DL, Thyer B. Does EMDR reduce post-traumatic stress disorder symptomatology in combat veterans? Behav Intervent. 2010;25:1-19. doi:10.1002/bin.295 